\title{
Referring Patients with Iron Deficiency Anaemia to Colorectal Surgery under the Two-Week Wait Rule
}

\author{
Mohammad Miah, Humayra Begum, Fraser Currie, Thomas Wild, Mohamad Al Saramigy \\ Cardiovascular and Thoracic National Institute of Cardiovascular Disease, Dhaka, Bangladesh \\ Email:mohammad.miah1@nhs.net
}

How to cite this paper: Miah, M., Begum, H., Currie, F., Wild, T. and Al Saramigy, M. (2020) Referring Patients with Iron Deficiency Anaemia to Colorectal Surgery under the Two-Week Wait Rule. Open Access Library Journal, 7: e6169.

https://doi.org/10.4236/oalib.1106169

Received: February 15, 2020

Accepted: March 17, 2020

Published: March 20, 2020

Copyright $\odot 2020$ by author(s) and Open Access Library Inc.

This work is licensed under the Creative Commons Attribution International License (CC BY 4.0).

http://creativecommons.org/licenses/by/4.0/

\begin{abstract}
Asymptomatic colonic and gastric carcinoma may present with IDA (Iron deficiency anaemia), and seeking these conditions is a priority in patients with IDA. One of the criteria under the Two-Week Referral (TWR) rule for suspected GI malignancy is iron deficiency anaemia (IDA). Although IDA is a good marker for gastrointestinal cancer, sometimes $2 \mathrm{WW}$ referral guidelines are not being followed appropriately for IDA. This not only has considerable workload and financial implications but also invasive investigations could lead to unnecessary adverse risks. Our aim was to find the proportion of patients referred as a $2 \mathrm{WW}$ for IDA not meeting the criteria, and the cost accrued by unnecessary referral. Also, to find out the cancer pick up rate in the patients who were referred appropriately according to the guideline and who were referred in appropriately without following the guidelines, Retrospective data collected. Consecutive 60 patients referred over 1 month from 9/07/2019 till 8/08/2019 as IDA were identified using the hospitals cancer database. The male and female patients were almost equal. Minimum age was 33 years and maximum age was 94 years. 3 patients were excluded due to incomplete data. Among 57 patients 42 were referred as true IDA whereas 15 (26\%) were referred without having true IDA. They underwent colonoscopy and OGD. 5 patients were diagnosed with different cancers. All of them were in true IDA group. Cancer pick up rate in false IDA group was $0 \%$. Finally, it can be said that the inappropriate referral has no advantage in cancer picking up rather increasing unnecessary health expenditure. Thus, the referring guidelines should be followed to decrease the unnecessary cost.
\end{abstract}

\section{Subject Areas}

Clinical Trials, Gastroenterology \& Hepatology, Translational Medicine 


\section{Keywords}

Iron Deficiency Anaemia, Colorectal Cancer, 2 Weeks Wait Referral

\section{Background}

Iron deficiency anaemia (IDA) occurs in $2 \%-5 \%$ of adult men and postmenopausal women in the developed world and it is a common cause of referral to surgeons and gastroenterologists (4\% - 13\% of referrals) [1]. While menstrual blood loss is the most common cause of IDA in premenopausal women, blood loss from the GI tract is the most common cause in adult men and postmenopausal women. Asymptomatic colonic and gastric carcinoma may present with IDA, and seeking these conditions is a priority in patients with IDA. One of the criteria under the Two-Week Referral (TWR) rule for suspected GI malignancy is iron deficiency anaemia (IDA). Although IDA is a good marker for gastrointestinal cancer, sometimes $2 \mathrm{WW}$ referral guidelines are not being followed appropriately for IDA. This not only has considerable workload and financial implications but also invasive investigations could lead to unnecessary adverse risks. The aim of the present study is to determine the percentage of patients referred inappropriately under the TWR as IDA and the cost accrued by unnecessary referral. New NICE recommendation for 2015:

$\square$ Refer people using a suspected cancer pathway referral (for an appointment within 2 weeks) for colorectal cancer if:

- They are aged 40 and over with unexplained weight loss and abdominal pain or

- They are aged 50 and over with unexplained rectal bleeding or

- They are aged 60 and over with:

- Iron-deficiency anaemia or

- Changes in their bowel habit

- Tests show occult blood in their faeces (new NICE recommendation for 2015).

Consider a suspected cancer pathway referral (for an appointment within 2 weeks) for colorectal cancer in adults aged under 50 with rectal bleeding and any of the following unexplained symptoms or findings:

- Abdominal pain

- Change in bowel habit

- Weight loss

- Iron-deficiency anaemia (new NICE recommendation for 2015).

Consider a suspected cancer pathway referral (for an appointment within 2 weeks) for colorectal cancer in people with a rectal or abdominal mass (new NICE recommendation for 2015).

O Offer testing for occult blood in faeces to assess for colorectal cancer in adults without rectal bleeding or have unexplained symptoms but do not meet the 
criteria for a suspected cancer pathway.

\section{Methods}

Data were collected retrospectively. Consecutive 60 patients who were referred over 1 month from 9/07/2019 till 8/08/2019 as IDA (Iron deficiency anaemia) were identified using the hospitals cancer database. We included all the suspected cancer referral from GP as a 2 week wait referral for IDA. We excluded any other suspected cancer referral rather than IDA. Age, Hb, MCV, Ferritin, PR bleed and cancer status were recorded for each patient.

Our aim was to find the proportion of patients referred as a 2WW for IDA not meeting the criteria [Table 1], and the cost accrued by unnecessary referral. Also to find out the cancer pick up rate in the patients who were referred appropriately according to the guideline and who were referred in appropriately without following the guidelines.

Iron Deficiency Anaemia (IDA) was defined as

Anaemia in male $(\mathrm{Hb}<130 \mathrm{~g} / \mathrm{L})$ or female $(\mathrm{Hb}<120 \mathrm{~g} / \mathrm{L})$ AND

- $\quad$ ferritin $<15 \mathrm{mcg} / \mathrm{L}$

- Low MCV $<80$ femtolitres

[Source: NICE Anaemia, iron deficiency Last revised in September 2018. https://cks.nice.org.uk/anaemia-iron-deficiency]

\section{Results}

Total 60 patient's data were collected among which 3 patients were excluded due to incomplete data. Thus, total 57 patients were included for data analysis. Mean age was 73.35 ( 33 y - 94 y). 28 Males and 29 were females. Only 9 patients had per rectal bleeding.; 14 patients had either change in bowel habit or weight loss or both and 4 of them had loss of appetite. Mean Hb level was 96 (71 - 132). Mean Ferritin was $52(4-276)$ and Mean MCV was 80.22 (63.8 - 103).

Cancer pick up rate in true IDA patients (42) $11.9 \%$ whereas Cancer pick up

Table 1. NICE recommendation for 2015: When should I refer a person with IDA for suspected colorectal cancer?

\begin{tabular}{ccc}
\hline Anaemia (iron-deficiency), age 60 and over & Colorectal & $\begin{array}{c}\text { Refer people using a suspected cancer } \\
\text { pathway referral (for an appointment } \\
\text { within 2 weeks) }\end{array}$ \\
$\begin{array}{c}\text { Anaemia (iron-deficiency, unexplained) with } \\
\text { rectal bleeding in adults age under 50 }\end{array}$ & Colorectal & $\begin{array}{c}\text { Consider a suspected cancer pathway } \\
\text { referral (for an appointment within } 2 \\
\text { weeks) }\end{array}$ \\
$\begin{array}{c}\text { Anaemia (iron-deficiency) without rectal } \\
\text { bleeding in adults age under } 60\end{array}$ & Colorectal & $\begin{array}{c}\text { Offer testing for occult blood in faeces } \\
\text { Anaemia (even in the absence of } \\
\text { iron-deficiency) without rectal bleeding, age } \\
60 \text { and over }\end{array}$ \\
& Colorectal & Offer testing for occult blood in faeces
\end{tabular}

[Source: Suspected cancer: recognition and referral, NICE guideline. Published: 23 June 2015. https://www.nice.org.uk/guidance/ng12]. 
rate in not true IDA patients (15) were $0 \%$. Total 5 cancers were identified, 1 of them underwent Rt Hemicolectomy, 1 Underwent Defunctioning Ileostomy, 1 underwent Chemotherapy, gastric carcinoma was referred to tertiary hospital and 1 for palliative.

As the outcome was satisfactory it was decided for no further action to be taken. However, this would be re-audited to see continuous improvement (Figures 1-6).

\section{Discussion}

These data demonstrate that anaemia with evidence of iron deficiency in patients referred via the $2 \mathrm{WW}$ pathway for suspected colorectal cancer (CRC) indicates an increase in the likelihood of CRC diagnosis compared with those who are having anaemia without iron deficiency. Clearly, this has value in deciding which cases are most likely to benefit from colonoscopy and OGD. IDA is amenable to therapy and this is particularly important as preoperative anaemia is strongly linked with higher morbidity and mortality following surgery. The cost of diagnostics in the $2 \mathrm{WW}$ pathway for CRC is large, and the majority of the

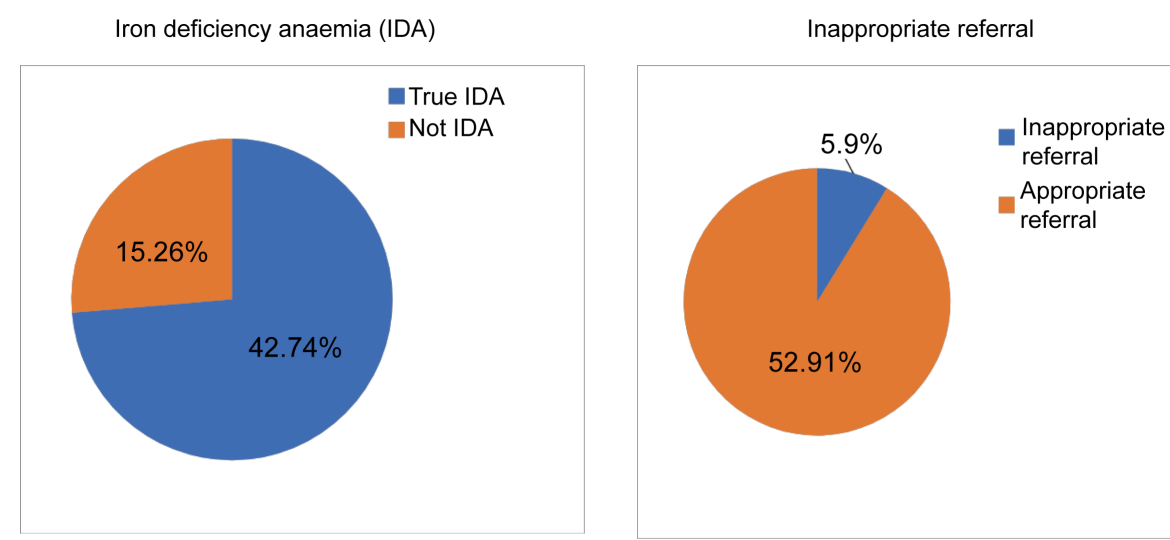

Figure 1. Pie charts showing IDA and inappropriate referral.

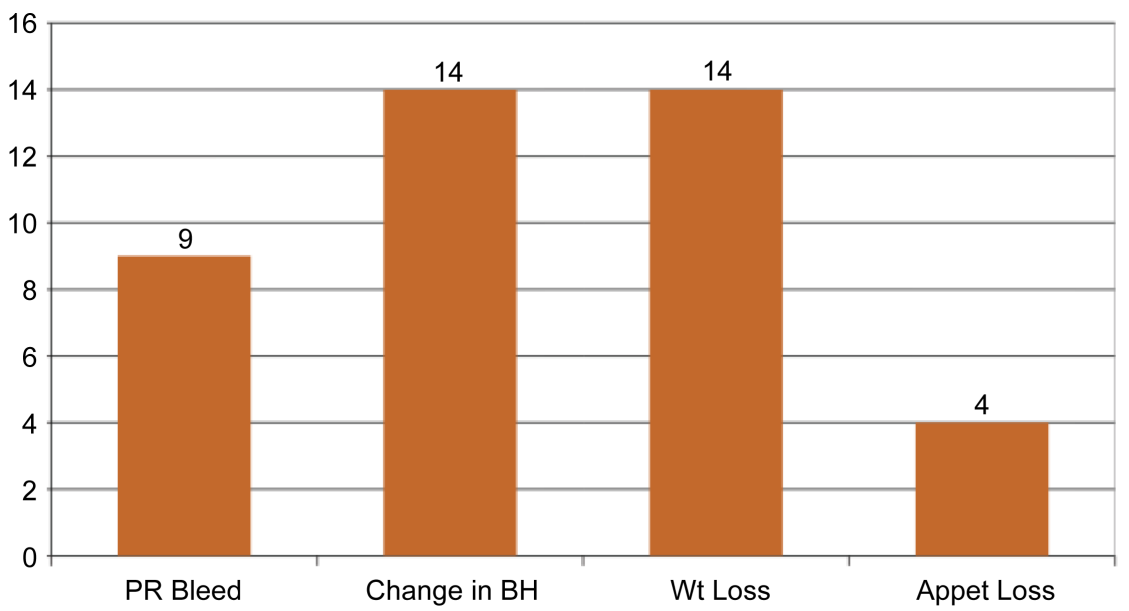

Figure 2. Bar graph showing different bowel symptoms in various number of patients. 


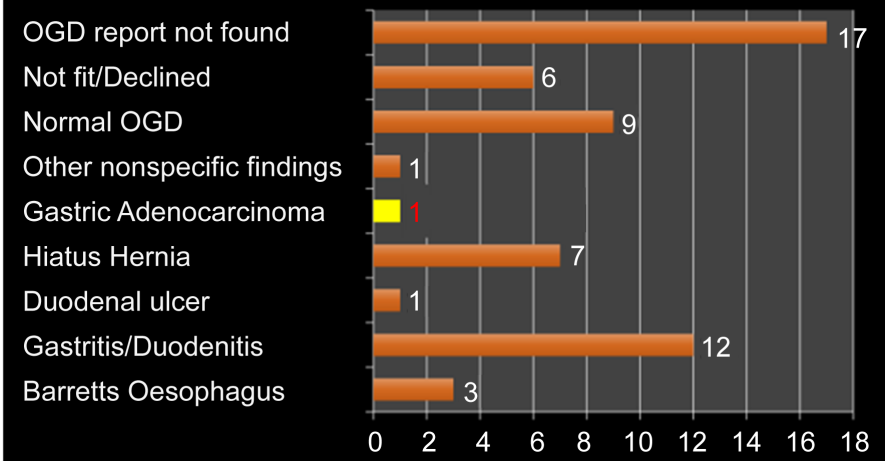

Figure 3. Showing various OGD findings in different patients.

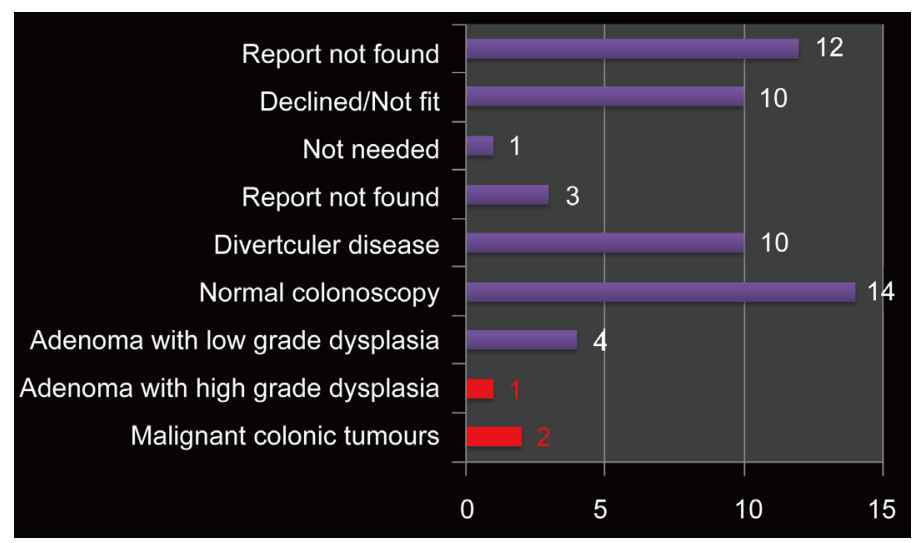

Figure 4. Bar graph showing colonoscopy findings in various patients.

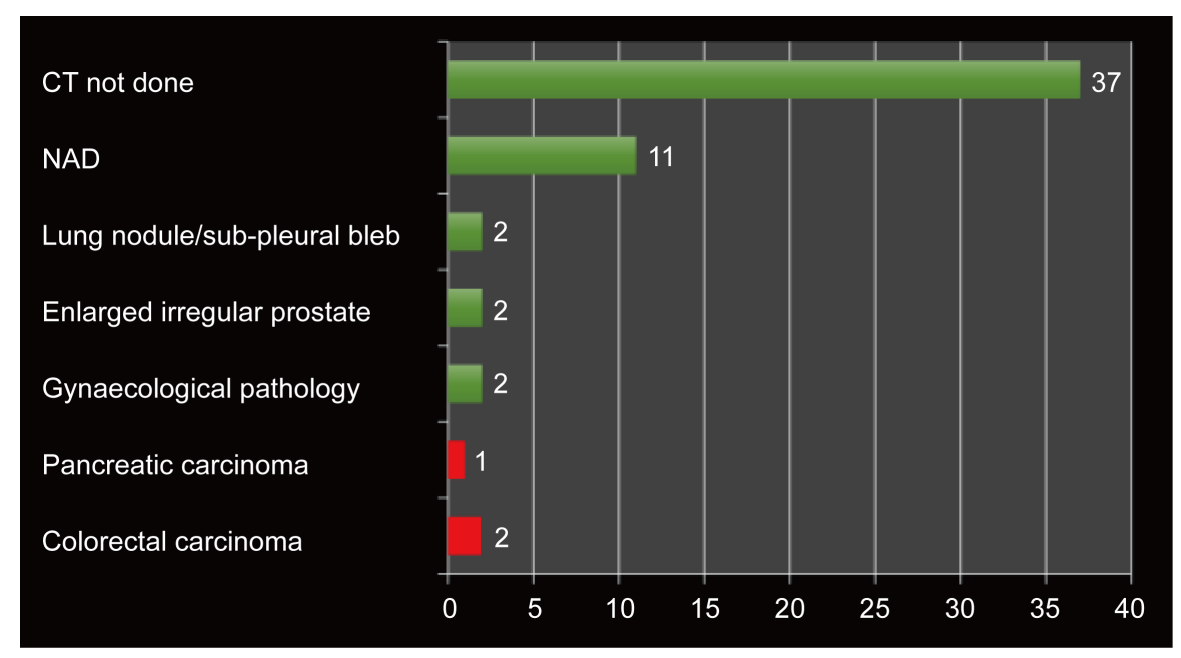

Figure 5. Bar graph showing CT scan findings in different patients.

patients suffer the anxiety and discomfort of invasive investigations without any treatable pathology identified. The value of IDA as an indicator for whole colon investigation as well as gastroscopy has been well described [1]. New NICE guidance on urgent cancer referral in June 2015 [2] broadened the range of cases to be referred and increased the pressure on secondary care services. Interestingly, they highlighted the significance of IDA in young patients with rectal 


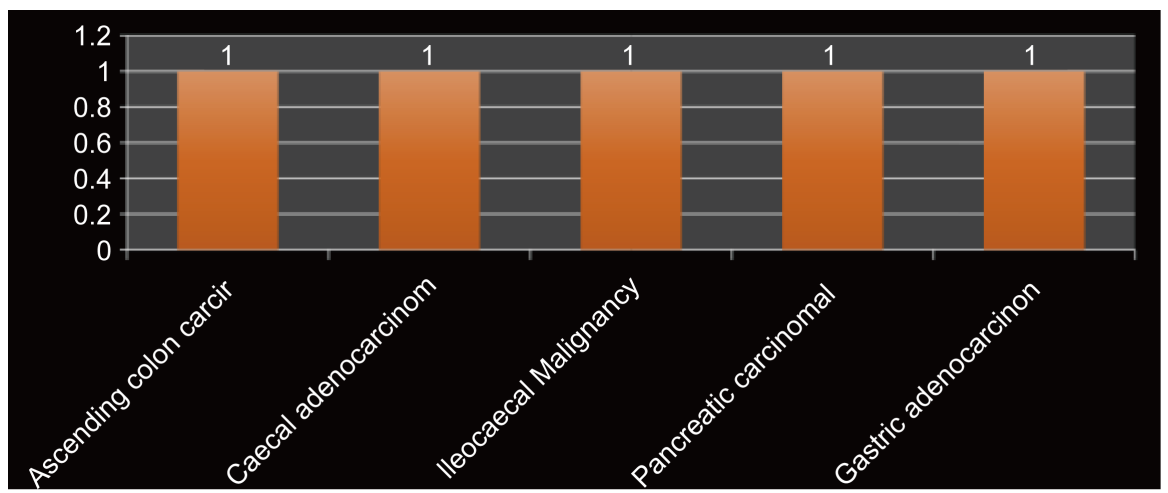

Figure 6. Showing Bar graph illustrating total number of cancers diagnosis. All were in true IDA group. No cancers were found in non-IDA group.

bleeding but not in patients with change in bowel habit. NICE also opened the door for faecal occult blood testing, including in patients aged $<60$ years.

Clearly, our 2WW pathway now aims to identify anaemia at referral in order to improve on both of these measures. Unfortunately, some of the referrals are still sent with no information on FBC (Full blood count). True IDA and strict referral criteria should be considered for every referral so that the clinical effectiveness of our $2 \mathrm{WW}$ pathway can be improved.

\section{Conclusion}

Although iron deficiency is a good marker for gastrointestinal cancer, it is evident that $2 \mathrm{WW}$ referral guidelines are not being followed. Referrals are still inappropriate according to the guidelines; however, the outcome was satisfactory. This inappropriate referral not only has considerable workload and financial implications but could be potentially detrimental to patient health.

\section{Conflicts of Interest}

There are no conflicts of interest to disclose. The project was registered with the clinical audit team of the hospital and was approved. Ethical approval was not required as the data was collected retrospectively and no intervention was done.

\section{References}

[1] Goddard, A.F., James, M.W., McIntyre, A.S. and Scott, B.B. (2011) Guidelines for the Management of Iron Deficiency Anaemia. Gut, 60, 1309-1316. https://doi.org/10.1136/gut.2010.228874

[2] National Institute for Health and Care Excellence (2015) Suspected Cancer: Recognition and Referral. London: NICE. 